\title{
Fatores prognósticos de letalidade hospitalar por diarréia ou pneumonia em menores de um ano de idade. Estudo de caso e controle*
}

\author{
Prognostic factors for hospital case-fatality due to diarrhea or pneumonia: a \\ case-control study
}

Cora Luiza Araújo Post"*, Cesar Gomes Victora***, Joaquim Gonçalves Valente****, Maria do Carmo Leal ${ }^{* * * *}$, Flávia Maria Leal Niobey****, Paulo Chagastelles Sabroza"***

\begin{abstract}
POST, C.L.A. et al. Fatores prognósticos de letalidade hospitalar por diarréia ou pneumonia em menores de um ano de idade. Estudo de caso e controle. Rev. Saúde públ.,S.Paulo, 26: 369- 78, 1992. Estudou-se a letalidade hospitalar devido à diarréia ou pneumonia em menores de um ano de idade na Região Metropolitana do Rio de Janeiro cujo 6́bito ocorreu entre abril/86 e maio/87. Foram investigados possíveis fatores prognósticos de letalidade hospitalar em relação a condições socioeconômicas, biológicas e amamentação aos 30 dias de vida. Foi utilizada metodologia de caso-controle, sendo casos as crianças internadas por diarréia ou por pneumonia que morreram, e controles aquelas que sobreviveram. Referências são feitas aos fenômenos de causalidade reversa e "overmatching" como possíveis viéses neste tipo de estudo. Razão de produtos cruzados (RPC) foi utilizada para estimar os riscos relativos, através de regressão logística não condicional. Os principais fatores prognósticos encontrados foram prematuridade, baixo peso ao nascer, mau estado geral e déficit peso/idade na hospitalização. Nas crianças com pneumonia a duração do aleitamento materno esteve associado com a letalidade $(R P C=2,0)$. As condições biológicas evidenciaram-se como os principais fatores prognósticos de letalidade hospitalar por diarréia ou pneumonia.
\end{abstract}

Descritores: Mortalidade infantil. Diarréia infantil, mortalidade. Pneumonia, mortalidade. Fatores de risco.

\section{Introduçăo}

A mortalidade infantil tem sido amplamente utilizada na avaliação do nível de saúde de uma determinada população, através de períodos caracterizados por diferentes conjunturas políticoeconômicas, por ser suficientemente sensível para refletir as agressões do meio ambiente em curto espaço de tempo ${ }^{2,3}$.

* Resumo da Dissertação de Mestrado apresentada à Esola Nacional de Saúde Pública, sob o título: "Fatores Prognósticos de Letalidade Hospitalar por Diarreia ou Pneumonia: um estudo de Caso e Controle", 1990. Apresentado no $1^{2}$ Congresso Brasileiro de Epidemiologia, Campinas, 1990. Financiado pela FINEP (Financiadora de Estudos e Projetos - Convênio n 43860197.0C).

** Departamento de Nutrição da Faculdade de Nutrição da Universidade Federal de Pelotas, RS - Brasil.

*** Centro de Pesquisas Epidemiológicas da Universidade Federal de Pelotas, RS.

**** Departamento de Epidemiologia e Métodos Quantitativos em Saúde da Escola Nacional de Saúde Pública da Fundação Oswaldo Cruz. Manguinhos - Rio de Janeiro, RJ - Brasil.

Separatas/Reprints: C.L.A. Post - Caixa Postal 354 - Campus Universitário - 96001-970 - Pelotas, RS Brasil
Vários estudos têm demonstrado, de diferentes formas, a determinação social da mortalidade infantil: Breilh e col. ${ }^{6}$, em Quito, Equador, em 1977; Guimarães e Fishmann ${ }^{13}$, em Porto Alegre, em 1980; Victora e Blank ${ }^{29}$, estudaram a mortalidade infantil segundo a estrutura agrária no Rio Grande do Sul; Monteiro e $\mathrm{col}^{18}$, no Município de São Paulo, em 1976; Pharoah e Morris ${ }^{21}$ estudaram a mortalidade infantil pós-neonatal segundo diferentes classificações socioeconômicas.

Embora ainda elevados em muitos países, estes índices vêm decrescendo na maior parte do mun$\mathrm{do}^{25}$. Este declínio nem sempre resultou de melhorias no poder de compra dos salários, qualidade de habitação e nível de emprego, mas possivelmente de intervençб̃es específicas no campo social e particularmente na saúde ${ }^{3}$, ou ainda de alteraçōes na estrutura de nascimentos com conseqüente redução no número de gestantes de maior risco (alta paridade, baixa ou alta idade e pouca escolaridade, por exemplo).

A mortalidade infantil por causas específicas também vem apresentando reduçōes importantes, especialmente as doenças intestinais e infecçōes respiratórias, o que explica em grande parte o decréscimo observado na mortalidade infantil geral, especialmente na pós-neonatal $2,3,22$. 
Outros estudos têm mostrado que, assim como para a mortalidade, são também as crianças de baixa renda as mais freqüentemente hospitalizadas, principalmente devido à diarréia e suas complicaçōes (desidratação, por exemplo) e às infecçð̃es respiratórias, especialmente a pneumonia ${ }^{1,28}$.

O presente estudo buscou investigar possíveis fatores prognósticos de letalidade hospitalar em crianças menores de um ano de idade residentes na Região Metropolitana do Rio de Janeiro, falecidas entre maio/86 e abril/87, tendo diarréia ou pneumonia como causa básica de óbito. Assim, pretende-se investigar, através de um estudo de casos e controles, alguns possíveis fatores socioeconômicos e biológicos que possam explicar o fato de que algumas crianças recuperam-se enquanto outras acabam morrendo. Com isto, pretende-se trazer novos subsídios que possam contribuir para a contínua queda das taxas de mortalidade infantil, especialmente por doenças para as quais se dispõe de amplo conhecimento técnico, como a diarréia e pneumonia.

\section{Material e Método}

A unidade de análise do estudo foi a Região Metropolitana do Rio de Janeiro (Rio de Janeiro, Nova Iguaçu, Duque de Caxias, Niterói, São João de Meriti, Nilópolis), excluídos alguns municípios de difícil acesso (Mangaratiba, Paracambi, Itaguaí, Magé, Itaboraí e Maricá). Foram incluídas as crianças residentes e falecidas dentro desta área de abrangência.

A hipótese do presente estudo era de que a letalidade hospitalar em crianças menores de um ano, internadas por diarréia ou pneumonia, seria mais elevada entre aquelas que apresentam uma ou mais das seguintes características: baixo nível socioeconômico, condições ambientais inadequadas, reduzido espaçamento interpartal, prematuridade, baixo peso ao nascer, desmame precoce, déficit de peso/idade na internação e grave estado geral no momento da hospitalização.

\section{Fonte de casos e seleção de controles}

Os atestados de óbito foram codificados pela Secretaria Estadual de Saúde do Rio de Janeiro, segundo a CID-98. O sorteio da amostra foi feito dentre os óbitos de menores de um ano de idade (6.337 óbitos), após estratificação por idade, de modo que a amostra contivesse $40 \%$ dos óbitos por todas as causas no período pós-neonatal e $15 \%$ no período neonatal. Dentre estes foram selecionados aqueles cuja causa básica de óbito fosse diarréia (CID 9-001 a 9-009) ou pneumonia (CID 9-480 a 9-487) ${ }^{8}$ e, excluindo-se aqueles que não pertenciam a área de estudo, obtiveram-se 635 óbitos, sendo que 448 (71\%) ocorreram no hospital e 187 (29\%) fora do hospital. Portanto, a amostra de casos compõe-se de 448 óbitos hospitalares, sendo 223 devido à diarréia e 225 à pneumonia (Fig.1).

Para cada óbito hospitalar devido a essas doenças tentou-se localizar uma criança internada no mesmo hospital, com a mesma doença e com diferença de idade inferior a dois meses, que tivesse sobrevivido, constituindo-se esta em controle.

\section{Coleta de Dados}

A coleta dos dados foi feita através de dois questionários. $\mathrm{O}$ questionário hospitalar foi utiliza-

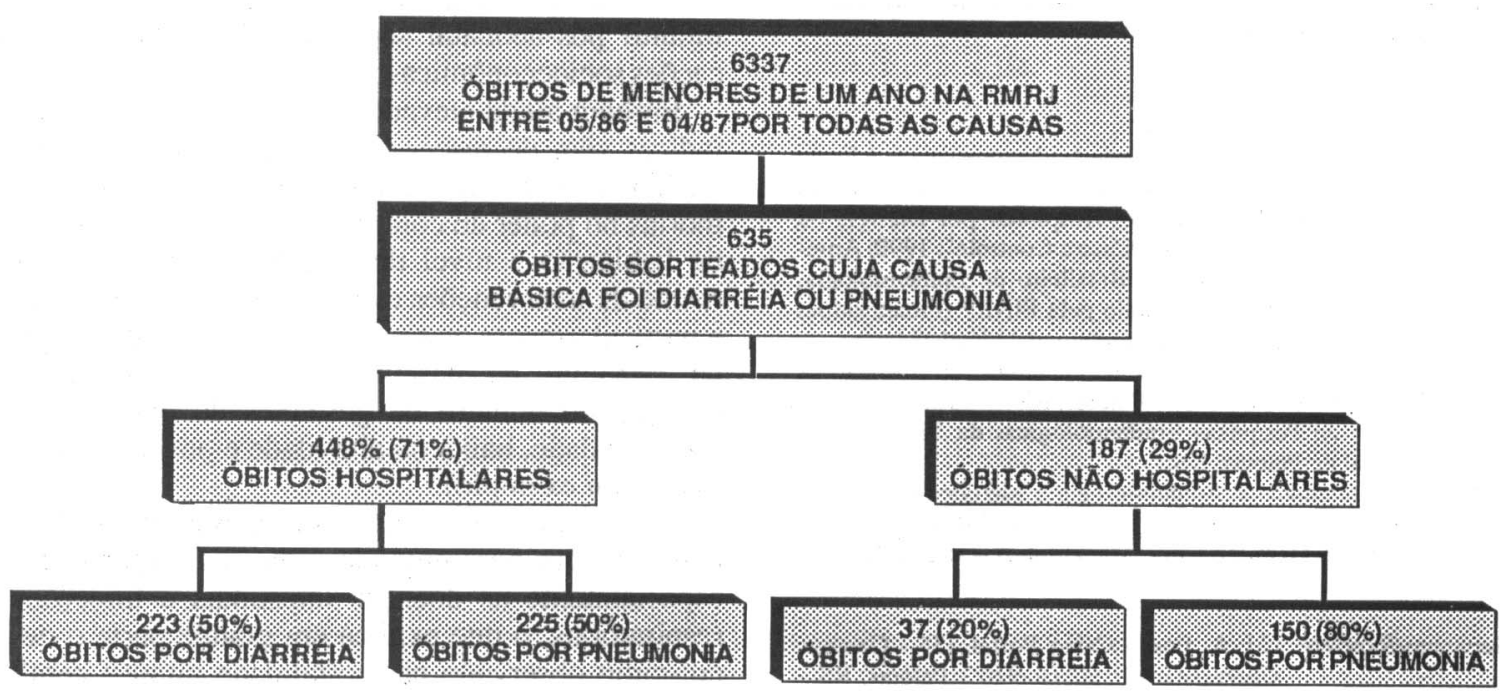

Figura 1. Composição da amostra de casos segundo a causa básica e o local de ocorrência do óbito. 
do para as informaçð̃es contidas no prontuário das crianças (casos e controles). Com o questionário domiciliar, aplicado à mãe ou responsável pela criança, obtiveram-se informaçoes socioeconômicas da familia e dados sobre a criança. Os dados hospitalares foram coletados por médicos enquanto as informaçð̃es domiciliares foram obtidas por entrevistadores com nível secundário de instrução. Ambos os grupos de entrevistadores receberam treinamento, com o objetivo de uniformizar os procedimentos.

As variáveis assim obtidas permitiram descrever algumas características da amostra (idade, sexo, outras complicaçð̃es presentes na internação de acordo com o responsável pela criança ou constatação médica), além de avaliar possiveis fatores prognósticos de letalidade hospitalar.

Como fatores prognósticos (Fig.2) entende-se aqueles que permitem predizer a evolução provável da doença, depois de instalada ${ }^{10}$. Portanto, no presente estudo, esperava-se que esses fatores estivessem diferencialmente distribuidos entre casos e controles. Foram os seguintes os fatores prognósticos estudados: renda familiar per capita, escolaridade da mãe, número de moradores por cômodo, tempo de gestação, intervalo interpartal, estado geral na hospitalização (obtido através do relato do exame físico da criança descrito no prontuário), estado de hidratação (presença ou ausência de desidratação, conforme dados do prontuário), peso ao nascer, relação peso/idade na internação (para comparação foi adotado o padrão do National Center for Health Statistics/NCHS ${ }^{19}$, amamentação aos 30 dias.

Por se tratar de estudo retrospectivo, não foi possível discriminar as crianças que foram desmamadas devido à doença que as levou à morte. As-

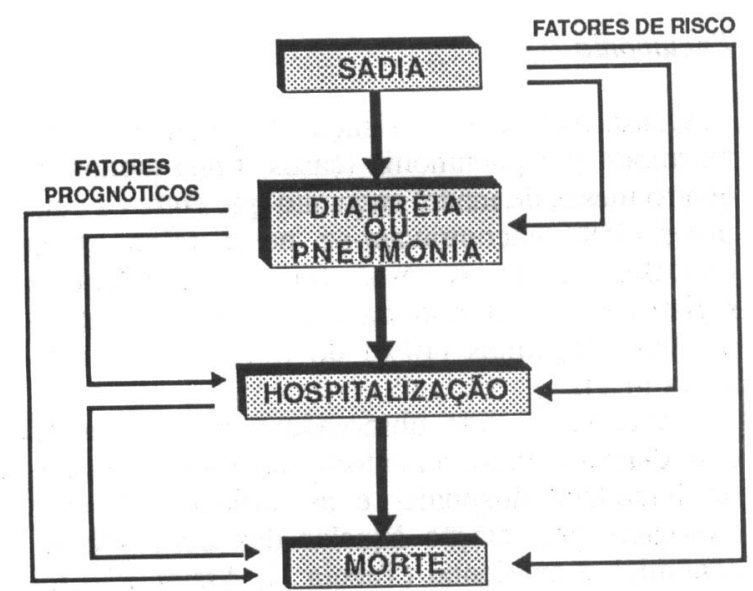

Figura 2. Esquema conceitual de fatores de risco e fatores prognóticos. sim, foram classificadas como desmamadas algumas crianças que, ao adoecerem, possivelmente ainda estivessem sendo amamentadas. Isso levaria a um aumento dos riscos relativos associados com o desmame, uma vez que o desmame devido à doença seria mais comum entre os casos do que entre os controles, dada a diferença na gravidade do quadro clínico. Para tentar evitar este viés foi utilizado um recurso metodológico recomendado por Habicht e col. ${ }^{14}$. Assim, análise foi restrita a crianças com idade igual ou superior a dois meses na época do óbito (casos) ou da internação (controles). Para estas, verificou-se se ainda estavam sendo amamentadas com um mês de vida. Dessa forma garantiu-se um intervalo de, pelo menos, 30 dias entre a data de referência para o desmame e o óbito ou internação, proporcionando alguma segurança de que estes não tenham sido o motivo da suspensão da amamentação.

\section{Emparelhamento}

Dos 448 óbitos hospitalares devidos à diarréia ou pneumonia, que deveriam ter sido investigados, foram efetivamente encontrados 404 (90\%) prontuários hospitalares. Destes, foram localizados os domicílios de 271 (60\%). Como um dos critérios de seleção do controle era de que este tivesse sido internado no mesmo hospital do caso, a não-localização do prontuário do caso implicou também perda de um possível controle (44 controles não puderam ser selecionados inicialmente). A Figura 1 apresenta o fluxograma de localização dos casos e controles bem como da obtenção das informações hospitalares e domiciliares.

\section{Análise estatística}

Para a análise estatística foi utilizada regressão logística* não condicional. Com esta técnica, foi calculada a razão de produtos cruzados (RPC ou "odds ratios") com intervalos de confiança a $95 \%$ (IC); a significância estatística foi avaliada pelo teste de razão de verossimilhança (para variáveis categóricas e para tendềncia linear). Apesar de na seleção ter sido adotado o emparelhamento, a análise foi realizada com os dados nãoemparelhados devido ao volume de perdas o que reduziria substancialmente o número de pares. Como a análise com dados não emparelhados tende a subestimar o risco, este seria, portanto, um viés conser-

Epidemiological Graphics, Estimation an Testing package - EGRET. Washington. Statistics and Epidemiology Research Corporation. 
vador. Para a análise, foram usados os programas CASP *, SPSS PC+ ** e EGRET ***.

Como a maior parte das variáveis estava disponível para, pelo menos, 100 casos e 100 controles, e considerando que $50 \%$ dos controles estudados estivessem expostos a um dos fatores prognósticos, o estudo teve um poder estatístico de $90 \%$ de detectar, como significativa, uma estimativa de risco igual ou superior a $2,55^{22}$.

\section{Resultados}

\section{Diarréia}

As idades das crianças, referentes a casos e controles, não apresentaram diferença estatisticamente significativa $(p=0,1)$. Entretanto, chama a atenção a predominância de crianças com pouca idade ( $79 \%$ dos casos e $76 \%$ dos controles tinham menos de seis meses). $O$ registro de outras infecções concomitantes foi significativamente $(p<0,001)$ mais freqüente nos prontuários dos casos $(39 \%)$ do que para os controles (21\%).

Em relação aos fatores prognósticos estudados (Tabela 1), a renda familiar per capita não se mostrou diferente entre casos e controles. Entretanto, cabe ressaltar que os dois grupos caracterizaram-se por pertencerem a famílias de baixa renda (aproximadamente $65 \%$ recebendo 0,5 salário-mínimo per capita, ou menos), sendo que apenas $8 \%$ dos casos e $10 \%$ dos controles possuiam renda per capita superior a um salário-mínimo. Tampouco houve diferença quanto à escolaridade materna, sendo que $13 \%$ das mães de casos e controles não eram alfabetizadas. Crianças que moravam em domicílios com quatro ou mais moradores por cômodo (28\% dos casos e $13 \%$ dos controles) apresentaram letalidade hospitalar 3,5 vezes maior do que aquelas residentes em casas com menos de dois moradores por cômodo, havendo uma relação linear entre aglomeração e letalidade.

O risco de óbito hospitalar foi quatro vezes maior para as crianças que nasceram com tempo de gestação igual ou inferior a 37 semanas $(p<0,001)$. Embora sem apresentar associação com a letalidade, chamou a atenção o percentual de intervalos interpartais curtos (menos de 24 meses) tanto entre casos (42\%) como entre os controles (39\%).

\footnotetext{
* Anthropometric Software Package - CASP. Atlanta. Center of Disease Control. Division of Nutrition.

** NIE, N. et al. SPSS: Statistical Package for the Social Sciences -24. ed. New York, McGraw-Hill, 1975.

*** Epidemiological Graphics, Estimation an Testing package - EGRET. Washington. Statistics and Epidemiology Research Corporation.
}

O estado geral com que as crianças chegaram ao hospital mostrou-se fortemente associado à letalidade $(p<0,001)$. A estimativa de risco relativo foi sete vezes maior para aquelas que apresentavam estado geral grave na internação. As crianças sem registro do estado geral no prontuário apresentaram letalidade intermediária $(R P C=1,7$ e IC $=1,0-2,8)$ em relação àquelas com estado moderado $(R P C=1,0)$ e grave ( $R P C=7,1$ e IC $=4,1-12,5)$.

$O$ peso ao nascer foi considerado outro importante fator prognóstico de letalidade hospitalar entre as crianças com diarréia. O risco de óbito foi três vezes maior $(I C=1,2-8,3)$ para as crianças que nasceram com peso entre zero e $2.500 \mathrm{~g}$, elevando-se para sete vezes $(\mathrm{IC}=2,1-23,9)$ entre os nascidos com menos de $2.000 \mathrm{~g}$ (Tab.1 e Fig.3). A relação peso/idade na internação também mostrou uma clara tendência de agravamento do prognóstico para as crianças mais desnutridas; a Tabela 1 mostra os resultados brutos e ajustados para o estado de hidratação da criança, através de regressão logística.

$O$ exame das estimativas de risco (RPC), mostrados na Tabela 1 e graficamente (em escala logarítmica) na Figura 3, mostram que a letalidade aproximadamente duplica com cada mudança de categoria do estado nutricional, embora a estimativa de risco (RPC) para as crianças com desnutrição severa (escore $Z \leq-3$ ) tenha sido três vezes superior ao da categoria imediatamente anterior. $O$ teste para tendência linear foi altamente significativo $(p<0,001)$ e não houve tendência de desvio de linearidade $(p>0,05)$.

Não foi encontrada associação entre amamentação e letalidade hospitalar por diarréia, sendo muito semelhantes as freqüências de amamentação até um mês de idade entre casos (60\%) e controles (59\%).

\section{Pneumonia}

Quase metade das crianças hospitalizadas que morreram por pneumonia (casos) tinha menos de quatro meses de idade, enquanto que entre os controles $35 \%$ encontravam-se nesta faixa etária $(p=0,008)$. Entre os casos, foi mais frequiente $o$ registro no prontuário da existência de malformaçōes congênitas (14\%) do que entre os controles (4\%).

Como havia sido observado para as crianças com diarréia, foram mais fortes as associaçōes entre letalidade hospitalar e as variáveis do tipo biológico, em relação àquelas dos tipos socioeconômico e ambiental (Tabela 1). Assim, não foi encontrada diferença significativa na renda per capita das familias de casos e controles sendo, entretanto, muito baixos os níveis de renda nos dois 
Tabela 1. Razáo de produtos cruzados (RPC) e teste para tendência linear de fatores prognóticos para letalidade hospitalar por diarréia entre crianças menores de um ano de idade residentes na Região Metropolitana do Rio de Janeiro. Maio/86 a abril/87.

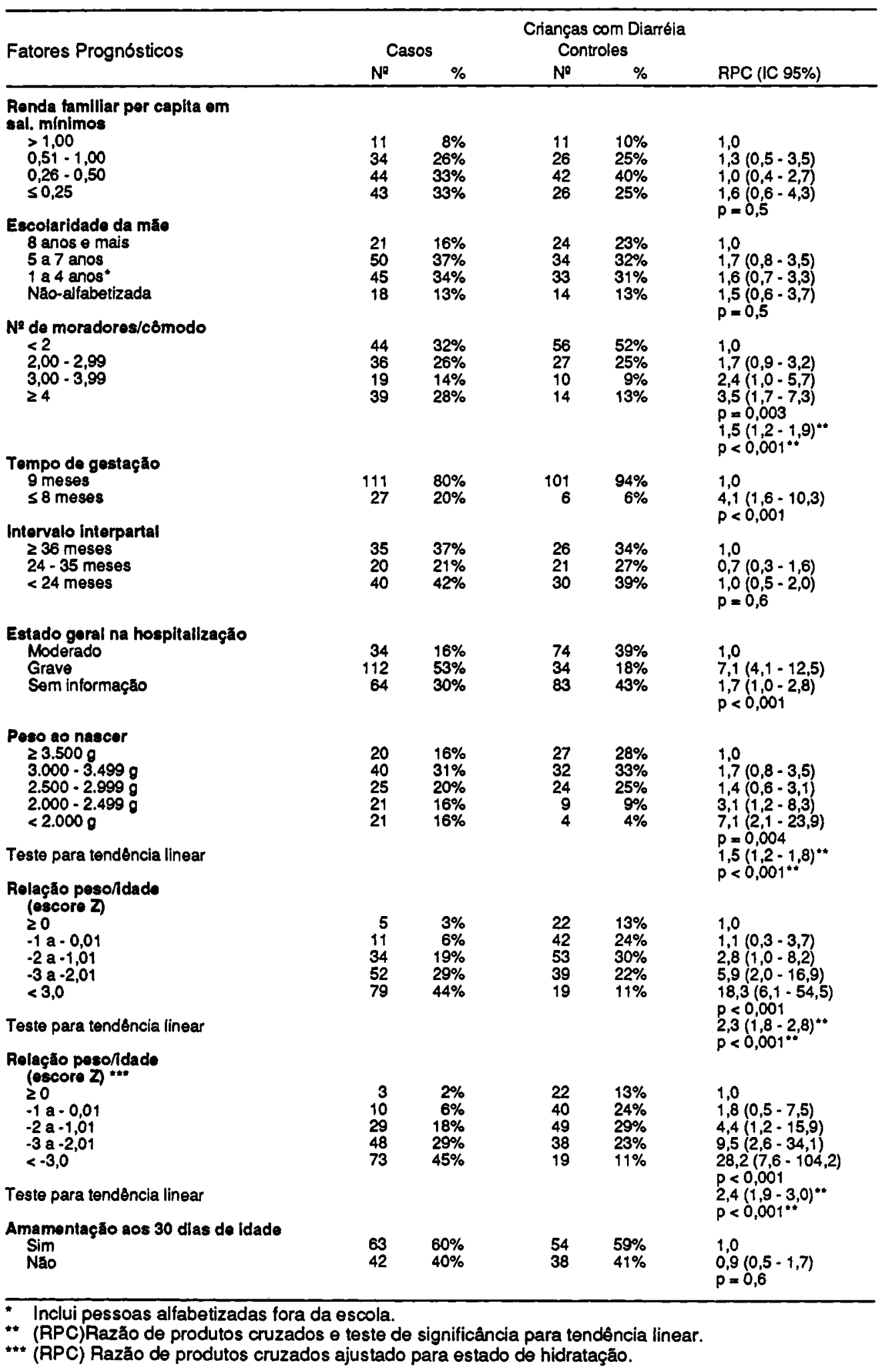


Tabela 2. Razão de produtos cruzados (RPC) e teste para tendéncia linear de fatores prognóticos para letalidade hospitalar por pneumonia entre crianças menores de um ano de idade residentes na Região Metropolitana do Rio de Janeiro. Maio/86 a abril/87.

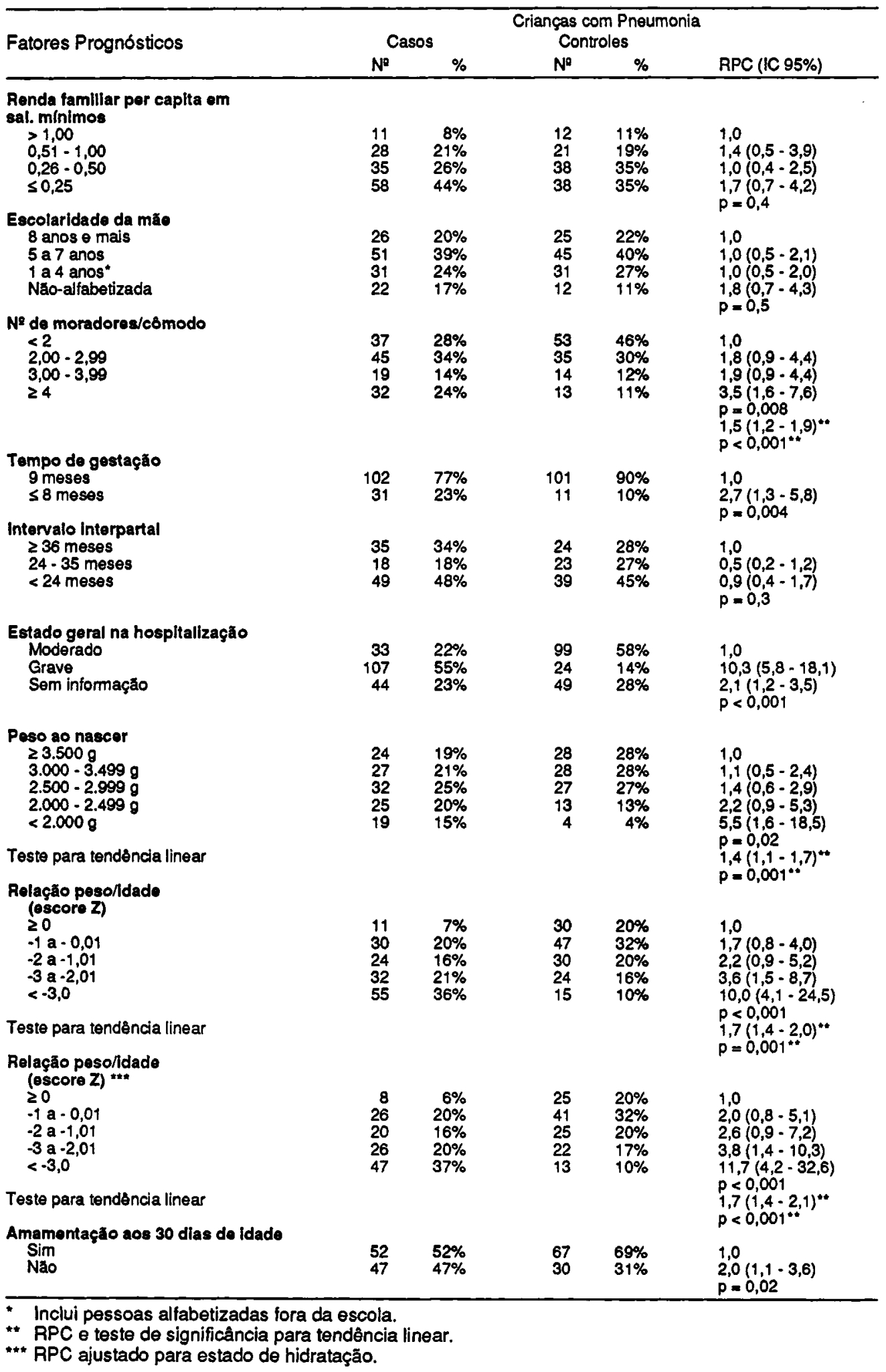




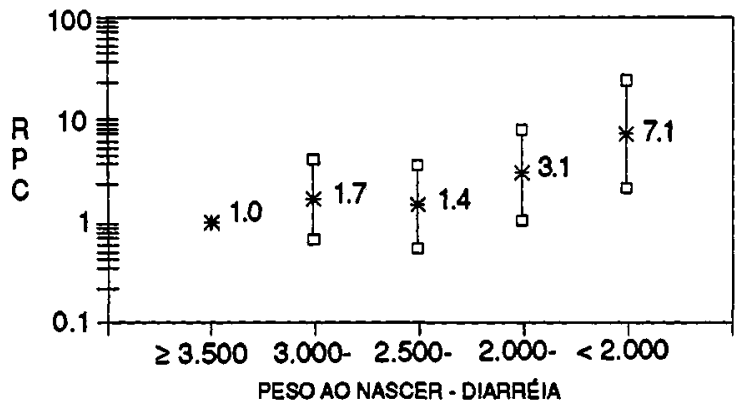

* RPC D INTERV CONF 95\%

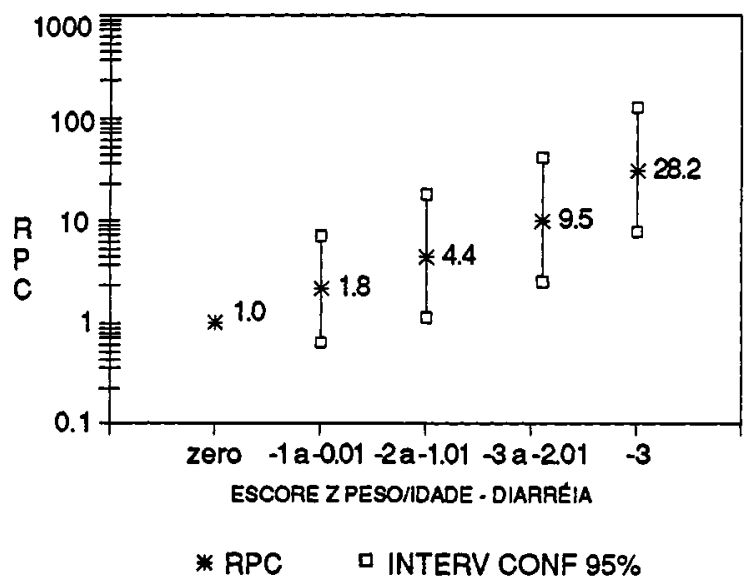

Figura 3. Razão de produtos cruzados (RPC) e intervalos de confiança para letalidade hospitalar por diarréia relativos ao peso ao nascer e escore $Z$ de peso/idade na internaçăo. Rio de Janeiro, maio/86 a abril/87.

grupos. Tampouco houve diferença em relação à escolaridade materna, sendo elevado em ambos os grupos o número de mães não-alfabetizadas ou com poucos anos de frequência à escola.

Quanto à aglomeração, 24\% dos casos e $11 \%$ dos controles, moravam em domicílios com quatro ou mais moradores por cômodo. A letalidade hospitalar foi 3,5 vezes maior para estas crianças do que para aquelas residentes em domicilios com menos de dois moradores por cômodo, havendo uma tendência linear significativa $(p<0,001)$.

As crianças cujo tempo de gestação foi igual ou inferior a 37 semanas apresentaram letalidade 2,7 vezes maior. Em relação ao estado geral da criança quando hospitalizada, aquelas em estado grave tiveram letalidade substancialmente maior ( $R P C=10,3$ e IC $=5,8-18,1 ; p<0,001$ ).

As variáveis antropométricas (peso ao nascer e relação peso/idade na internação) estiveram também significativamente associadas com a letali-

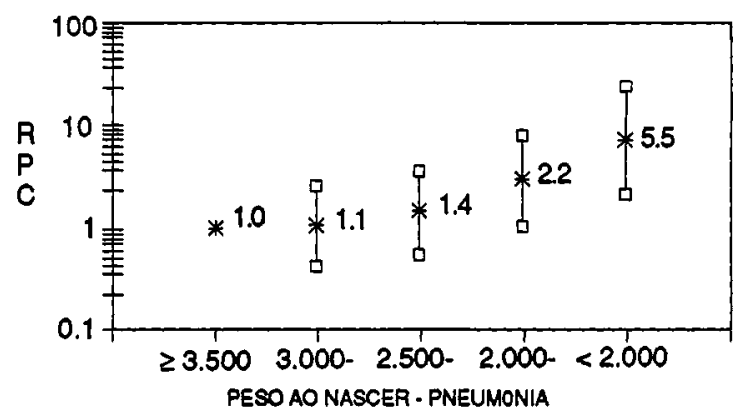

* RPC $\quad$ INTERV CONF 95\%

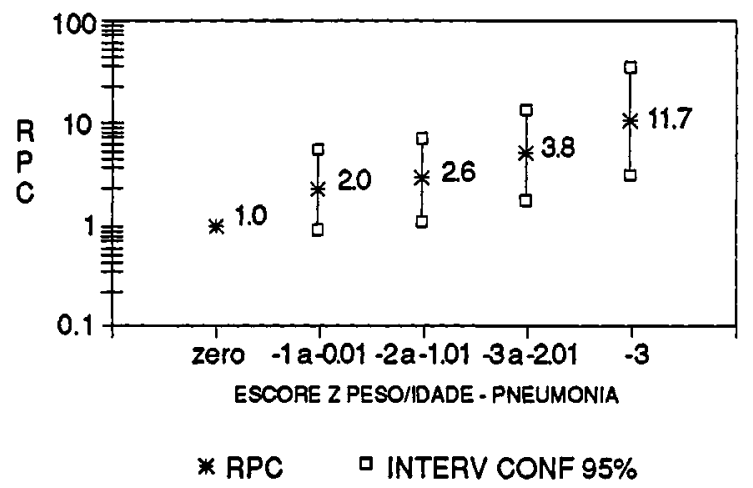

Figura 4. Razão de produtos cruzados (RPC) $\theta$ intervalos de confiança para letalidade hospitalar por pneumonia relativos ao peso ao nascer e escore $Z$ de pesofidade na internação. Rio de Janeiro, maio/86 a abril/87.

dade por pneumonia $(p<0,001)$. Entre os casos, $35 \%$ das crianças tiveram peso de nascimento inferior a $2.500 \mathrm{~g}$, enquanto que entre os controles apenas 17\% (Tabela 2 e Fig.4) o que indica uma letalidade hospitalar de aproximadamente 2,5 vezes maior para as crianças nascidas com baixo peso. A relação entre peso/idade e letalidade não foi tão forte quanto para a diarréia. $O$ grupo de desnutridos severos (escore $Z \leq-3$ ) apresentou letalidade três vezes superior à da categoria adjacente (Tabela $2 \mathrm{e}$ Fig.4). O teste para tendência linear, no entanto, foi altamente significativo $(p<0,001)$ e não houve evidência estatística de desvio de linearidade (p>0,05).

Em relação à amamentação observou-se que as crianças desmamadas apresentaram letalidade duas vezes superior à das crianças amamentadas $(\mathrm{IC}=1,1-3,6)$.

\section{Discussăo e Conclusóes}

A maior letalidade por pneumonia (mas não por diarréia) observada entre recém-nascidos pode ser 
constatada, apesar do emparelhamento por idade $( \pm 2$ meses). Os casos com diarréia apresentaram semelhante distribuição etária em relação aos controles.

Dentre os fatores prognósticos investigados, a renda familiar per capita e a escolaridade matema não apresentaram diferenças estatisticamente significativas entre casos e controles (Tabelas 1 e 2). Isto pode ser explicado pela restriçäo do estudo a crianças hospitalizadas com diarréia ou pneumonia, as quais, em sua quase totalidade, provém de famílias de baixo nível socioeconômico. Este fenômeno $\varepsilon$ análogo àquele conhecido como sobre-emparelhamento (ou "overmatching")22, em que casos e controles resultam muito semelhantes por haverem sido emparelhados por variáveis relacionadas com os fatores de risco em estudo. Outra possível explicação seria o fato de que não foi feita análise com dados emparelhados, o que pode ter prejudicado a detecção de associação. Chama ainda a atenção o fato de mais de $10 \%$ das crianças estudadas serem filhas de mães analfabetas, apesar de residirem em área urbana, onde teoricamente haveria melhores oportunidades para a freqüência a escolas. Por outro lado, pode ser que estas mães tenham migrado para esta região após a idade usual de alfabetização, não tendo tido nova oportunidade para frequientar a escola.

O número de moradores por cômodo apresentou associação significativa com a letalidade. Como indicador de condição socioeconômica, pode ter-se mostrado mais sensível que os demais indicadores; como indicador de aglomeração, por si sugere ser um fator de risco de infecção por determinados agentes, os quais apresentam maior letalidade (rotavirus, por exemplo) do que agentes menos intensamente relacionados com aglomeração (por exemplo, bactérias fecais) ${ }^{4,11}$. O teste de tendência linear mostrou que há uma relação do tipo dose-resposta, isto $\hat{e}$, quanto maior a aglomeração maior a letalidade.

Entre as crianças prematuras (nascidas com menos de 37 semanas de gestação) a maior letalidade está parcialmente mediada pelo baixo peso ao nascer. Além disso, os prematuros também podem apresentar problemas relativos a imaturidade pulmonar, o que pode contribuir para o aumento da mortalidade por pneumonia. Como a maior parte das crianças estudadas tinha menos de seis meses, é possivel que esse tempo não tenha sido suficiente para superar estas desvantagens para sua sobrevivência, situação esta agravada por outras dificuldades devido a condiçð̄es ambientais e socioeconômicas adversas. Este resultado coincide com os achados do estudo longitudinal de Pelo$\operatorname{tas}^{28}$, onde a mortalidade entre os prematuros foi cerca de onze vezes maior do que entre os nascidos a termo com peso adequado.
Embora óbvio, o grave estado geral com que a criança chega ao hospital, apresentou-se como um importante fator prognóstico de letalidade hospitalar. Alguns possíveis mecanismos podem ser sugeridos para explicar este achado: a) a família, especialmente a mãe, não percebe a gravidade do episódio e não busca ajuda. Resultados semelhantes foram encontrados em outro estudo no Rio de Janeiro, em relação a desidratação, principalmente entre as mães com menor escolaridade ${ }^{16} ; b$ ) a familia busca ajuda mas não a consegue (pouca disponibilidade de serviços, filas, horários de atendimento, entre outros); c) a criança chega ao médico mas este não avalia corretamente a gravidade do quadro e não a encaminha para hospitalização; d) a criança tem outros fatores subjacentes (infecções repetidas, baixo peso ao nascer, desnutrição, e outros) que fazem com que o quadro piore rapidamente. Estes possíveis mecanismos podem atuar isoladamente ou em conjunto. Estudos mais detalhados seriam necessários para esclarecer as razões pelas quais os casos eram internados em piores condições físicas.

Outros indicadores que se mostraram importantes fatores prognósticos foram o peso ao nascer e o peso/idade da criança na internação. Em relação a este último, o peso ao nascer tem a vantagem de não apresentar os inconvenientes causados pelo fenômeno de causalidade reversa 14,26,27,30, pelo qual a relação peso/idade na internação, ao invés de ser um fator de risco para a letalidade, seria de fato o resultado de uma doença de maior severidade, o qual poderia levar a perda de massa corporal. Outro fenômeno que poderia causar baixo peso na admissão é a presença de desidratação ${ }^{4}$; no entanto, os resultados acima citados, sobre a relação entre peso/idade e letalidade, foram previamente ajustados para o estado de hidratação (Tabelas $1 \mathrm{e} 2$ ).

Em resumo, não é plausível explicar a fortíssima associação encontrada no presente estudo apenas em termos de causalidade reversa ou de diferenças entre casos e controles quanto ao grau de desidratação. Além disso, outros estudos mostram que também a relação altura/idade de crianças hospitalizadas devido a diarréia ou pneumonia apresenta-se comprometida ${ }^{27}$, sugerindo que também o déficit ponderal pode ter sido anterior ao inicio da doença. Esta idéia é reforçada pelos presentes resultados relativos ao peso ao nascer e à relação entre peso/idade e pneumonia, a qual provoca menor perda de massa corporal do que a diarréia ${ }^{20}$.

Embora vários estudos tenham proporcionado razoável conhecimento sobre a relação entre desnutrição e infecção ${ }^{5,12,23}$, uma questão ainda aberta refere-se aos níveis de mortalidade de crianças 
com diferentes graus de desnutrição. Diversos autores têm analisado se 0 aumento da mortalidade é gradativo (linear) ou se há um efeito do tipo limiar ("threshold") $5,12,15,24,27$. À primeira vista, os resultados do atual estudo parecem estar em concordância com estudos de $\mathrm{Chen}^{7}$ e Martorell ${ }^{17}$, quando descrevem $o$ efeito de tipo limiar ("threshold") segundo o qual a partir de um determinado ponto de corte ocorre um intenso aumento na estimativa de risco (RPC), indicando uma piora brusca no prognóstico. Este fenômeno parece ocorrer também em relação ao peso ao nascer (Tabelas 1 e 2). No entanto, para este assim como para o indicador peso/idade os intervalos de confiança das estimativas do risco relativo (RPC) foram muito amplos; os testes de tendência linear resultaram significativos, o que não ocorreu com os testes de desvio de linearidade. Desta forma, não se pode concluir com segurança que tal efeito, tipo limiar ("threshold"), esteja presente.

A amamentação aos 30 dias apresentou associação significativa apenas com a letalidade por pneumonia. A redução do risco de óbito por pneumonia entre as crianças amamentadas (mesmo que por curto período) está de acordo com os resultados de Victora e col. ${ }^{30}$ segundo os quais a mortalidade infantil por pneumonia foi 3,6 vezes superior entre as crianças totalmente desmamadas. Deve-se considerar ainda a raridade do aleitamento exclusivo no País, mesmo em rescém-nascidos, o que contribui para reduzir o efeito protetor do leite materno, especialmente na prevenção ou recuperação da diarréia ${ }^{9}$.

A magnitude das perdas ocorridas no presente estudo, ainda que provavelmente não comprometa os achados mais importantes, recomenda cautela na análise dos resultados.

Em resumo, tanto casos quanto controles eram originários de famílias de estratos sociais baixos, não havendo no presente estudo associação entre fatores socioeconômicos e letalidade. A única exceção foi a forte associação linear encontrada com o número de moradores por cômodo, a qual pode ser possivelmente explicada pelo risco de contágio.

Já os fatores biológicos, dentre os quais destacam-se o tempo de gestação, o estado geral na hospitalização e os pesos ao nascer e na internação, mostraram-se sensiveis indicadores da letalidade hospitalar. Merece maior discussão o fato de que os fatores "biológicos" parecem estar mais fortemente associados com a letalidade do que os fatores "sociais". Em primeiro lugar, todos os fatores biológicos estudados são em grande parte determinados pela situação social da família. Em segundo lugar, o objeto do estudo foi a letalidade hospitalar, e, portanto, para ser incluído como caso ou controle a criança necessitava ser hospitalizada com diarréia ou pneumonia. Sugerimos que os fatores sociais seriam de grande importância na determinação da incidência da doença e em sua gravidade (risco de hospitalização). Já entre aquelas crianças hospitalizadas, os maiores riscos estariam associados a características próprias da criança em nivel biológico, dado que o tratamento seria igual para todas.

Dentre os fatores identificados, especial atenção deveria ser dada à condição nutricional e ao estado geral em que as crianças são hospitalizadas. Medidas preventivas deveriam ser intensificadas em relação a gestantes e a crianças de alto risco sob o aspecto nutricional. Além disso, os profissionais da rede ambulatorial devem redobrar sua atenção para que essas crianças sejam identificadas, prontamente tratadas e, se necessário, internadas antes de evoluírem para um quadro clínico de difícil reversão.

POST, C.L.A. et al. [Prognostic factors for hospital case-fatality due to diarrhea or pneumonia: a casecontrol study]. Rev. Saúde públ., S.Paulo, 26: 369-78, 1992. Diarrhea and pneumonia are common diseases in children aged under one year, for which there are simple therapeutic measures. However, infant mortality due to these diseases is still very high, varying markedly according to socio-economic status. The characteristics of children who died (cases) and of those who were hospitalized with diarrhea or pneumonia, but survived (controls), were studied. The following groups of variables were studied: socio-economic, environmental and biological conditions, nutritional status and breast-feeding. Information on cases and controls was collected from hospital records and through home interviews. Important losses occurred in the latter: $40 \%$ of cases and $50 \%$ of controls were not interviewed. There were no significant differences between cases who were included and those who were not, in terms of age, sex or place of residence. To estimate relative risks of prognostic factors unconditional Logistic Regression was used to calculate the odds ratios and their $95 \%$ confidence intervals. Prematurity, low birth weight, weight/age deficit, presence of edema and poor general status at hospital admission were prognostic factors for hospital case-fatality. In relation to the anthropometric variables, it was not possible to conclude for certain whether the increased casefatality was linearly or non-linearly (threshold) associated with nutritional deficit. The duration of breastfeeding was only associated with case-fatality for pneumonia. Socio-economic factors were not important for the prognosis of children admitted to hospital with diarrhea or pneumonia. Some of the expected risk factor associations were not detected, maybe due to the small sample size (resulting from the high losses) which was insufficient to show small differences. In this study the biological conditions of children with diarrhea or pneumonia appeared to be the important prognostic factors for hospital case-fatality. 
Keywords: Infant mortality. Diarrhea infantile, mortality. Pneumonia, mortality. Risks factor.

\section{Referéncias Bibllográflcas}

1. BARROS, M.B.A. Morbidade e mortalidade hospitalar de criancas menores de um ano, em Ribeirão Preto, SP (Brasil), 1975. Rev. Saúde públ., S.Paulo, 15: 308-20, 1981.

2. BECKER, R.A. \& LECHTIG, A. Brasil: evoluçäo da mortalidade infantil no período 1977-1984. Brastlia. Ministério da Saúde, Divisão Nacional de Epidemiologia. 1986.

3. BIXBY, L.R. Determinantes del descenso de la mortalidade infantil en Costa Rica. Bol. Ofic. sanit. panamer., 99: 510-27,1985.

4. BLACK, R.E. Diarrheal diseases and child morbidity and mortality. Popul.Develop.Rev., 10(Suppl.): 141-61, 1984.

5. BLACK, R.E. et al. Malnutrition is a detemining factor in diartheal duration, but not incidence, among young children in a longitudinal study in rural Bangladesh. Amer. J. clin.Nutr., 37: 87-94, 1984.

6. BREILH, J. et al. Ciudad y muerte infantil. CEAS Ediciones, 1983.

7. CHEN, L.C. et al. Anthropometric assessment of energyprotein malnutrition and subsequent risk of mortality among preschool aged children. Amer.J. clin. Nutr.,33: $1836-45,1980$.

8. CLASSIFICAÇÃO Intermacional de Doenças; 9a. revisão, 1975. São Paulo, Organizaçăo Mundial da Saúde/ Centro da OMS para Classificação de Doenças em Português, 1978.

9. FEACHEM, R.G. \& KOBLINSKY, M.A. Interventions for the control of diarthoeal diseases among young children: promotion of breast-feeding. Bull. wld Hlih Org. 62:271-91,1984.

10. FLETCHER, R.H. et al. Prognóstico. In: Fletcher, R.H. et al. Epidemiologia clínica. Porto Alegre, Artes Médicas, 1989. p. 145-72.

11. GARDNER, G. a al. Effects of social and family factors on viral respiratory infection and illness in the first year of life. J. Epidem. community Hlth, 38: 42-8, 1984.

12. GOMEZ, F. Desnutritión. Bol. med. Hosp. inf. México, 3(4) 1946.

13. GUIMARĀES, J.J.L. \& FISHNANN, A. Desigualdades na mortalidade infantil entre favelados e não favelados no município de Porto Alegre, Rio Grande do Sul, em 1980. Bol. Ofic. sanit. panamer., 101: 19-35, 1986.

14. HABICHT, J.P. et al. Does breastfeeding really save lives, or are apparent benefits due to biases? Amer. J. Epidem.,123: 279-90, 1986.

15. KIELMANN, A.A.\& MCCORD, C. Weight-for-age as an index of risk of death in children. Lancet, 1:1247-50, 1978.
16. LEAL, M.C. et al Percepção matema da desidratação em crianças com diarretia: estudo de concordância com diagnóstico médico. Rev. Saúde públ., S.Paulo, 24: 196203, 1990.

17. MARTORELL, R. \& HO, T.J. Malnutrition morbity, and mortality. Popul. Develop. Rev., 10 (Suppl.): 49-68, 1984.

18. MONTEIRO, C.A. et al. Mortalidade no primeiro ano de vida e a distribuição de renda e de recursos públicos de saúde, São Paulo (Brasil). Rev. Saúde públ., S.Paulo 14: 515-39, 1980.

19. NCHS growth curves for children, birth-18 year. United States. Vital Hlth Slatist. Ser. 11, (165) 1978.

20. ORGANIZACION PANAMERICANA DE LA SALUD. Manual de tratamiento de la diarreia. Washington, 1987. (Serie PALTEX para executores de programas de salud, $\left.n^{2} 13\right)$.

21. PHAROAH, P.O.D. \& MORRIS, J.N. Postneonatal mortality. Epidem. Rev., 1: 170-83, 1979.

22. SCHLESSELMAN, J.J. Case-control studies: design, conduct. analysis. New York, Oxford University Press, 1982.

23. SCRIMSHAW, N.S. A al. Nutricion y infecciones: su accion reciproca. Ginebra, Organizacion Mundial de la Salud, 1970. (Série de monografias $n^{2} 57$ ).

24. TOMKINS A.M. et al. Nutritional status and risk of morbity among young Gambian children allowing for social and environmental factors. Trans. roy. Soc. trop. Med. Hyg., 83: 282-7, 1989.

25. UNICEF. Situação mundial da infância - 1990. Brasilia, 1990.

26. VICTORA, C.G. Case-control studies of influence of breastfeeding on child morbity and mortality: methodological issues. In: Atkinson, S.A. et al., eds. Breastfeeding nutrition, infection and infant growth in developed an developing countries. St. John's, Canada, ARTS Biomedical Publ., 1989.

27. VICTORA, C.G. et al. Pneumonia, dianthea and growth in the first four years of life: a longitudinal study of 5,914 urban Brazilian children. Amer. J. clin. Nutr. (no prelo).

28. VICTORA, C.G. at al. Epidemiologia da desigualdade: um estudo longitudinal de 6.000 crianças brasileiras. São Paulo, Editora Hucitec, 1988.

29. VICTORA, C.G. \& BLANK, N. Mortalidade infantil e estrutura agrária no Rio Grande do Sul. Ciênc. e Cult., 32: 1223-33, 1980.

30. VICTORA, C.G. et al. Evidence for protection by breastfeeding against infant deaths from infectious diseases in Brazil. Lancet, 1: 319-22, 1987.

Recebido para publicaçäo em 19.12.1991

Reapresentado em 24.7.1992

Aprovado para publicaçäo em 1.9.1992 